Supplement of

\title{
Straw incorporation increases crop yield and soil organic carbon sequestration but varies under different natural conditions and farming practices in China: a system
} 5 analysis

Xiao Han ${ }^{1 \ddagger}$, Cong Xu ${ }^{1 \ddagger}$, Jennifer A. J. Dungait ${ }^{2}$, Roland Bol ${ }^{3}$, Xiaojie Wang ${ }^{1}$, Wenliang $\mathrm{Wu}^{1}$, and Fanqiao Meng ${ }^{1}$

${ }^{1}$ Beijing Key Laboratory of Biodiversity and Organic Farming, College of Resources and Environmental Sciences, China Agricultural University, Beijing, 100193, China

$10{ }^{2}$ Sustainable Agriculture Sciences, Rothamsted Research, North Wyke, Okehampton, Devon EX20 2SB, UK

${ }^{3}$ Institute of Bio- and Geosciences, Agrosphere (IBG-3), Forschungszentrum Jülich GmbH, 52425 Jülich, Germany

${ }^{\ddagger}$ These authors contributed equally to this work

15 Correspondence to: Fanqiao Meng (mengfq@cau.edu.cn) 
Table S1: The collected 68 publications used in this meta-analysis.

\begin{tabular}{|c|c|c|c|c|c|c|c|c|c|c|c|c|c|c|c|}
\hline No & Reference & Region & Coordinate & $\begin{array}{l}\text { Duration } \\
\text { (years) }\end{array}$ & MAT & MAP & Soil texture & $\begin{array}{l}\text { Initial } \\
\mathrm{SOC}(\mathrm{g} \\
\left.\mathrm{kg}^{-1}\right)\end{array}$ & $\begin{array}{l}\text { Land } \\
\text { use }\end{array}$ & $\begin{array}{l}\text { Crop } \\
\text { type }\end{array}$ & $\begin{array}{l}\text { Crop } \\
\text { frequency }\end{array}$ & $\begin{array}{l}\text { Straw } \\
\text { type }\end{array}$ & STC & NF & Yield \\
\hline 1 & $\begin{array}{l}\text { (Aiziguli-Mulati et } \\
\text { al., 2012) }\end{array}$ & NWC & $108.8^{\circ} \mathrm{N}, 35.13^{\circ} \mathrm{E}$ & 5 & 13 & 600 & Loam & 10.21 & $\mathrm{U}$ & $\mathrm{W}, \mathrm{M}$ & $\mathrm{D}$ & Maize & 0.62 & 330 & $\mathrm{Na}$ \\
\hline $2-1$ & (Bi et al., 2009) & $\mathrm{SC}$ & $116.17^{\circ} \mathrm{N}, 28.35^{\circ} \mathrm{E}$ & 26 & 18.1 & 1727 & Silt Loam & 16.2 & $\mathrm{P}$ & $\mathrm{R}$ & $\mathrm{D}$ & Rice & $\mathrm{Na}$ & 140 & $\mathrm{Y}$ \\
\hline $2-2$ & (Bi et al., 2009) & $\mathrm{SC}$ & $116.17^{\circ} \mathrm{N}, 28.35^{\circ} \mathrm{E}$ & 26 & 18.1 & 1727 & Silt Loam & 16.2 & $\mathrm{P}$ & $\mathrm{R}$ & $\mathrm{D}$ & Rice & $\mathrm{Na}$ & 140 & $\mathrm{Y}$ \\
\hline 3 & (Cai et al., 2011) & NWC & $106.5^{\circ} \mathrm{N}, 34.25^{\circ} \mathrm{E}$ & 10 & 10.5 & 538.2 & loam & 6.59 & $\mathrm{U}$ & M & $\mathrm{S}$ & Maize & 4.44 & 239 & $\mathrm{Y}$ \\
\hline $4-1$ & (Cao et al., 1995) & $\mathrm{SC}$ & $120.72^{\circ} \mathrm{N}, 31.59^{\circ} \mathrm{E}$ & 3 & 16.7 & 1241 & Clay & 20.83 & $\mathrm{P}-\mathrm{U}$ & $\mathrm{W}, \mathrm{R}$ & $\mathrm{D}$ & Rice & 0.95 & 120 & $\mathrm{Y}$ \\
\hline $4-2$ & (Cao et al., 1995) & $\mathrm{SC}$ & $120.72^{\circ} \mathrm{N}, 31.59^{\circ} \mathrm{E}$ & 3 & 16.7 & 1241 & Clay & 20.83 & $\mathrm{P}-\mathrm{U}$ & $\mathrm{W}, \mathrm{R}$ & $\mathrm{D}$ & Rice & 1.89 & 120 & $\mathrm{Y}$ \\
\hline 5 & (Chen et al., 2010) & $\mathrm{SC}$ & $120.71^{\circ} \mathrm{N}, 31.56^{\circ} \mathrm{E}$ & 12 & 16.7 & 1241 & Clay & 12.76 & P-U & $\mathrm{W}, \mathrm{R}$ & $\mathrm{D}$ & $\begin{array}{l}\text { Rice- } \\
\text { Wheat }\end{array}$ & 0.92 & 300 & $\mathrm{Na}$ \\
\hline $6-1$ & (Chen et al., 2009) & $\mathrm{SC}$ & $113.15^{\circ} \mathrm{N}, 29.37^{\circ} \mathrm{E}$ & 19 & 17.01 & 1568 & Sandy loam & 11.3 & $\mathrm{P}$ & $\mathrm{R}$ & $\mathrm{T}$ & Rice & $\mathrm{Na}$ & 0 & $\mathrm{Na}$ \\
\hline $6-2$ & (Chen et al., 2009) & $\mathrm{SC}$ & $112.56^{\circ} \mathrm{N}, 28.27^{\circ} \mathrm{E}$ & 19 & 17.2 & 1553 & Sandy loam & 17.1 & $\mathrm{P}$ & $\mathrm{R}$ & $\mathrm{T}$ & Rice & $\mathrm{Na}$ & 0 & $\mathrm{Na}$ \\
\hline $6-3$ & (Chen et al., 2009) & $\mathrm{SC}$ & $112.16^{\circ} \mathrm{N}, 28.54^{\circ} \mathrm{E}$ & 19 & 16.7 & 1681 & Loam & 19.4 & $\mathrm{P}$ & $\mathrm{R}$ & $\mathrm{T}$ & Rice & $\mathrm{Na}$ & 0 & $\mathrm{Na}$ \\
\hline $7-1$ & (Dong et al., 2012) & NWC & $102.6^{\circ} \mathrm{N}, 38.09^{\circ} \mathrm{E}$ & 20 & 7.7 & 150 & Loam & 9.49 & $\mathrm{U}$ & $\mathrm{W}, \mathrm{M}$ & $\mathrm{D}$ & $\begin{array}{l}\text { Wheat- } \\
\text { Maize }\end{array}$ & 3.53 & 0 & $\mathrm{Na}$ \\
\hline $7-2$ & (Dong et al., 2012) & NWC & $102.6^{\circ} \mathrm{N}, 38.09^{\circ} \mathrm{E}$ & 20 & 7.7 & 150 & Loam & 9.49 & $\mathrm{U}$ & $\mathrm{W}, \mathrm{M}$ & $\mathrm{D}$ & $\begin{array}{l}\text { Wheat- } \\
\text { Maize }\end{array}$ & 1.77 & 188 & $\mathrm{Na}$ \\
\hline 8 & (Dong et al., 2013) & NEC & $124.24^{\circ} \mathrm{N}, 43.32^{\circ} \mathrm{E}$ & 5 & 5.8 & 577 & Clay & 11.69 & $\mathrm{U}$ & M & $\mathrm{S}$ & Maize & 1.26 & 252 & $\mathrm{Na}$ \\
\hline $9-1$ & (Fang et al., 2003) & NWC & $110.12^{\circ} \mathrm{N}, 35.34^{\circ} \mathrm{E}$ & 10 & 11.5 & 553 & Silt Loam & 6.38 & $\mathrm{U}$ & $\mathrm{W}$ & $\mathrm{S}$ & Maize & 1.26 & 165 & $\mathrm{Y}$ \\
\hline $9-2$ & (Fang et al., 2003) & NWC & $110.12^{\circ} \mathrm{N}, 35.34^{\circ} \mathrm{E}$ & 10 & 11.5 & 553 & Silt Loam & 6.38 & $\mathrm{U}$ & $\mathrm{W}$ & $\mathrm{S}$ & Wheat & 2.52 & 165 & $\mathrm{Y}$ \\
\hline $10-1$ & (Gao et al., 2012) & $\mathrm{NC}$ & $115.05^{\circ} \mathrm{N}, 35.69^{\circ} \mathrm{E}$ & 6 & 14.52 & 627.5 & Sandy loam & 8.93 & $\mathrm{U}$ & $\mathrm{W}, \mathrm{M}$ & $\mathrm{D}$ & $\begin{array}{l}\text { Wheat- } \\
\text { Maize }\end{array}$ & $\mathrm{Na}$ & 450 & $\mathrm{Na}$ \\
\hline $10-2$ & (Gao et al., 2012) & $\mathrm{NC}$ & $115.05^{\circ} \mathrm{N}, 35.69^{\circ} \mathrm{E}$ & 6 & 14.52 & 627.5 & Sandy loam & 8.93 & $\mathrm{U}$ & $\mathrm{W}, \mathrm{M}$ & $\mathrm{D}$ & Wheat & $\mathrm{Na}$ & 450 & $\mathrm{Na}$ \\
\hline $10-3$ & (Gao et al., 2012) & $\mathrm{NC}$ & $115.05^{\circ} \mathrm{N}, 35.69^{\circ} \mathrm{E}$ & 6 & 14.52 & 627.5 & Sandy loam & 8.93 & $\mathrm{U}$ & $\mathrm{W}, \mathrm{M}$ & $\mathrm{D}$ & Maize & $\mathrm{Na}$ & 450 & $\mathrm{Na}$ \\
\hline $11-1$ & (Gao et al., 2000) & $\mathrm{SC}$ & $120.65^{\circ} \mathrm{N}, 31.86^{\circ} \mathrm{E}$ & 12 & 15.2 & 1039.3 & Clay & 11.43 & P-U & $\mathrm{W}, \mathrm{R}$ & $\mathrm{D}$ & $\begin{array}{l}\text { Rice- } \\
\text { Wheat }\end{array}$ & 3.52 & 338 & $\mathrm{Na}$ \\
\hline $11-2$ & (Gao et al., 2000) & $\mathrm{SC}$ & $120.65^{\circ} \mathrm{N}, 31.86^{\circ} \mathrm{E}$ & 12 & 15.2 & 1039.3 & Clay & 11.43 & P-U & $\mathrm{W}, \mathrm{R}$ & $\mathrm{D}$ & $\begin{array}{l}\text { Rice- } \\
\text { Wheat }\end{array}$ & 3.2 & 338 & $\mathrm{Na}$ \\
\hline 12 & $(\mathrm{Gao}, 2013)$ & NEC & $126.37^{\circ} \mathrm{N}, 45.57^{\circ} \mathrm{E}$ & 3 & 5 & 500 & loam & 12.65 & $\mathrm{U}$ & M & $\mathrm{S}$ & Maize & 3 & 0 & $\mathrm{Na}$ \\
\hline 13 & (Gao et al., 2011) & NEC & $126.48^{\circ} \mathrm{N}, 43.97^{\circ} \mathrm{E}$ & 16 & 5.6 & 562.8 & Clay & 13.14 & $\mathrm{U}$ & M & $\mathrm{S}$ & Maize & $\mathrm{Na}$ & 112 & $\mathrm{Y}$ \\
\hline 14 & (Gu et al., 2004) & NWC & $108.08^{\circ} \mathrm{N}, 34.3^{\circ} \mathrm{E}$ & 12 & 13 & 632 & Silt Loam & 6.4 & $\mathrm{U}$ & $\mathrm{W}, \mathrm{M}$ & $\mathrm{D}$ & Maize & 2 & 312 & $\mathrm{Y}$ \\
\hline $15-1$ & (Guan et al., 2014) & NWC & $104.61^{\circ} \mathrm{N}, 35.57^{\circ} \mathrm{E}$ & 11 & 6.4 & 390.9 & Silt Loam & 6.97 & $\mathrm{U}$ & $\mathrm{W}$ & $\mathrm{S}$ & Multiple & $\mathrm{Na}$ & 63 & $\mathrm{Na}$ \\
\hline
\end{tabular}




\begin{tabular}{|c|c|c|c|c|c|c|c|c|c|c|c|c|c|c|c|}
\hline $15-2$ & (Guan et al., 2014) & NWC & $104.61^{\circ} \mathrm{N}, 35.57^{\circ} \mathrm{E}$ & 11 & 6.4 & 390.9 & Silt Loam & 6.97 & $\mathrm{U}$ & $\mathrm{W}$ & $\mathrm{S}$ & Multiple & $\mathrm{Na}$ & 63 & $\mathrm{Na}$ \\
\hline $15-3$ & (Guan et al., 2014) & NWC & $104.61^{\circ} \mathrm{N}, 35.57^{\circ} \mathrm{E}$ & 11 & 6.4 & 390.9 & Silt Loam & 6.97 & $\mathrm{U}$ & $\mathrm{W}$ & $\mathrm{S}$ & Multiple & $\mathrm{Na}$ & 63 & $\mathrm{Na}$ \\
\hline $15-4$ & (Guan et al., 2014) & NWC & $104.61^{\circ} \mathrm{N}, 35.57^{\circ} \mathrm{E}$ & 11 & 6.4 & 390.9 & Silt Loam & 6.97 & $\mathrm{U}$ & W & $\mathrm{S}$ & Multiple & $\mathrm{Na}$ & 63 & $\mathrm{Na}$ \\
\hline $16-1$ & (Guo et al., 2003) & $\mathrm{NC}$ & $115.23^{\circ} \mathrm{N}, 37.94^{\circ} \mathrm{E}$ & 10 & 12 & 488 & Loam & 5.05 & $\mathrm{U}$ & $\mathrm{W}, \mathrm{M}$ & $\mathrm{D}$ & Wheat & 1.53 & 150 & $\mathrm{Y}$ \\
\hline $16-2$ & (Guo et al., 2003) & $\mathrm{NC}$ & $115.23^{\circ} \mathrm{N}, 37.94^{\circ} \mathrm{E}$ & 10 & 12 & 488 & Loam & 5.05 & $\mathrm{U}$ & $\mathrm{W}, \mathrm{M}$ & $\mathrm{D}$ & Wheat & 1.87 & 150 & $\mathrm{Y}$ \\
\hline 17 & (Han et al., 2010) & $\mathrm{NC}$ & $114.71^{\circ} \mathrm{N}, 37.9^{\circ} \mathrm{E}$ & 7 & 12.5 & 480.7 & Loam & 9.3 & $\mathrm{U}$ & $\mathrm{W}, \mathrm{M}$ & $\mathrm{D}$ & Maize & $\mathrm{Na}$ & 439 & $\mathrm{Na}$ \\
\hline 18 & (Hao et al., 2013) & NEC & $126.92^{\circ} \mathrm{N}, 47.45^{\circ} \mathrm{E}$ & 8 & 1.5 & 550 & Clay & 27.54 & $\mathrm{U}$ & M & $\mathrm{S}$ & Multiple & 4.81 & 150 & $\mathrm{Na}$ \\
\hline 19 & (He and $\mathrm{Ni}, 1996)$ & $\mathrm{SC}$ & $119.18^{\circ} \mathrm{N}, 29.04^{\circ} \mathrm{E}$ & 4 & 17.1 & 1602.6 & Clay & 23.2 & $\mathrm{P}-\mathrm{U}$ & $\mathrm{W}, \mathrm{R}$ & $\mathrm{T}$ & Rice & 2.2 & 414 & $\mathrm{Y}$ \\
\hline 20 & (Hou et al., 2004) & NEC & $121.78^{\circ} \mathrm{N}, 42.15^{\circ} \mathrm{E}$ & 4 & 8.1 & 502 & Clay & 20.8 & $\mathrm{U}$ & M & $\mathrm{S}$ & Maize & 3.33 & 375 & $\mathrm{Na}$ \\
\hline 21 & (Huang, 1984) & $\mathrm{NC}$ & $116.8^{\circ} \mathrm{N}, 35.51^{\circ} \mathrm{E}$ & 5 & 13.6 & 733 & Clay & 6.38 & $\mathrm{U}$ & $\mathrm{W}, \mathrm{M}$ & $\mathrm{D}$ & Wheat & 3.08 & 318 & $\mathrm{Y}$ \\
\hline $22-1$ & $\begin{array}{l}\text { (Kong and } \mathrm{He} \text {, } \\
2003 \text { ) }\end{array}$ & $\mathrm{SC}$ & $116.94^{\circ} \mathrm{N}, 28.23^{\circ} \mathrm{E}$ & 13 & 17.6 & 1788.8 & Clay & 3.72 & $\mathrm{U}$ & $\mathrm{R}$ & $\mathrm{D}$ & Rice & 1.26 & 88 & $\mathrm{Na}$ \\
\hline $22-2$ & $\begin{array}{l}\text { (Kong and } \mathrm{He} \text {, } \\
2003 \text { ) }\end{array}$ & $\mathrm{SC}$ & $116.94^{\circ} \mathrm{N}, 28.23^{\circ} \mathrm{E}$ & 13 & 17.6 & 1788.8 & Clay & 3.72 & $\mathrm{U}$ & $\mathrm{R}$ & $\mathrm{D}$ & Multiple & 3.15 & 88 & $\mathrm{Na}$ \\
\hline 23 & $\begin{array}{l}\text { (Li et al., 1999); (Li } \\
\text { et al., 2007) }\end{array}$ & $\mathrm{NC}$ & $115.72^{\circ} \mathrm{N}, 37.73^{\circ} \mathrm{E}$ & 19 & 13 & 550 & Clay & 6.27 & $\mathrm{U}$ & $\mathrm{W}, \mathrm{M}$ & $\mathrm{D}$ & Wheat & 0.6 & 0 & $\mathrm{Y}$ \\
\hline $24-1$ & (Li et al., 2014) & $\mathrm{NC}$ & $116.56^{\circ} \mathrm{N}, 33.17^{\circ} \mathrm{E}$ & 5 & 14.8 & 821 & Clay & 8.26 & $\mathrm{U}$ & $\mathrm{W}, \mathrm{M}$ & $\mathrm{D}$ & $\begin{array}{l}\text { Wheat- } \\
\text { Maize }\end{array}$ & 4.1 & 0 & $\mathrm{Na}$ \\
\hline $24-2$ & (Li et al., 2014) & $\mathrm{NC}$ & $116.56^{\circ} \mathrm{N}, 33.17^{\circ} \mathrm{E}$ & 5 & 14.8 & 821 & Clay & 8.26 & $\mathrm{U}$ & $\mathrm{W}, \mathrm{M}$ & $\mathrm{D}$ & $\begin{array}{l}\text { Wheat- } \\
\text { Maize }\end{array}$ & 4.1 & 360 & $\mathrm{Na}$ \\
\hline $24-3$ & (Li et al., 2014) & $\mathrm{NC}$ & $116.56^{\circ} \mathrm{N}, 33.17^{\circ} \mathrm{E}$ & 5 & 14.8 & 821 & Clay & 8.26 & $\mathrm{U}$ & $\mathrm{W}, \mathrm{M}$ & $\mathrm{D}$ & $\begin{array}{l}\text { Wheat- } \\
\text { Maize }\end{array}$ & 4.1 & 450 & $\mathrm{Na}$ \\
\hline $24-4$ & (Li et al., 2014) & $\mathrm{NC}$ & $116.56^{\circ} \mathrm{N}, 33.17^{\circ} \mathrm{E}$ & 5 & 14.8 & 821 & Clay & 8.26 & $\mathrm{U}$ & $\mathrm{W}, \mathrm{M}$ & $\mathrm{D}$ & $\begin{array}{l}\text { Wheat- } \\
\text { Maize }\end{array}$ & 4.1 & 540 & $\mathrm{Na}$ \\
\hline $24-5$ & (Li et al., 2014) & $\mathrm{NC}$ & $116.56^{\circ} \mathrm{N}, 33.17^{\circ} \mathrm{E}$ & 5 & 14.8 & 821 & Clay & 8.26 & $\mathrm{U}$ & $\mathrm{W}, \mathrm{M}$ & $\mathrm{D}$ & $\begin{array}{l}\text { Wheat- } \\
\text { Maize }\end{array}$ & 4.1 & 630 & $\mathrm{Na}$ \\
\hline $24-6$ & (Li et al., 2014) & $\mathrm{NC}$ & $116.56^{\circ} \mathrm{N}, 33.17^{\circ} \mathrm{E}$ & 5 & 14.8 & 821 & Clay & 8.26 & $\mathrm{U}$ & $\mathrm{W}, \mathrm{M}$ & $\mathrm{D}$ & $\begin{array}{l}\text { Wheat- } \\
\text { Maize }\end{array}$ & 4.1 & 720 & $\mathrm{Na}$ \\
\hline 25 & (Li et al., 2009) & $\mathrm{SC}$ & $111.5^{\circ} \mathrm{N}, 28.92^{\circ} \mathrm{E}$ & 6 & 16.8 & 1230 & Loam & 7.89 & $\mathrm{P}-\mathrm{U}$ & $\mathrm{R}, \mathrm{M}$ & $\mathrm{D}$ & Rice & 2.12 & 280 & $\mathrm{Y}$ \\
\hline $26-1$ & (Li et al., 2006) & $\mathrm{SC}$ & $113.15^{\circ} \mathrm{N}, 29.37^{\circ} \mathrm{E}$ & 18 & 17.01 & 1568 & Sandy loam & 19.44 & $\mathrm{P}-\mathrm{U}$ & $\mathrm{R}$ & $\mathrm{T}$ & Rice & $\mathrm{Na}$ & 0 & $\mathrm{Na}$ \\
\hline $26-2$ & (Li et al., 2006) & $\mathrm{SC}$ & $112.56^{\circ} \mathrm{N}, 28.27^{\circ} \mathrm{E}$ & 18 & 17.2 & 1553 & Sandy loam & 17.05 & $\mathrm{P}-\mathrm{U}$ & $\mathrm{R}, \mathrm{M}$ & $\mathrm{T}$ & $\begin{array}{l}\text { Rice- } \\
\text { Wheat }\end{array}$ & $\mathrm{Na}$ & 0 & $\mathrm{Na}$ \\
\hline $26-3$ & (Li et al., 2006) & $\mathrm{SC}$ & $113.16^{\circ} \mathrm{N}, 27.72^{\circ} \mathrm{E}$ & 18 & 17.6 & 1525 & Clay & 23.2 & P-U & $\mathrm{R}$ & $\mathrm{T}$ & Rice & $\mathrm{Na}$ & 0 & $\mathrm{Na}$ \\
\hline $26-4$ & (Li et al., 2006) & $\mathrm{SC}$ & $112.16^{\circ} \mathrm{N}, 28.54^{\circ} \mathrm{E}$ & 18 & 16.7 & 1681 & Clay & 23.61 & P-U & $\mathrm{R}, \mathrm{M}$ & $\mathrm{T}$ & $\begin{array}{l}\text { Rice- } \\
\text { Wheat }\end{array}$ & $\mathrm{Na}$ & 0 & $\mathrm{Na}$ \\
\hline $26-5$ & (Li et al., 2006) & $\mathrm{SC}$ & $112.16^{\circ} \mathrm{N}, 28.54^{\circ} \mathrm{E}$ & 18 & 16.7 & 1419 & Clay & 32.08 & $\mathrm{P}-\mathrm{U}$ & $\mathrm{R}$ & $\mathrm{T}$ & Rice & $\mathrm{Na}$ & 0 & $\mathrm{Na}$ \\
\hline $27-1$ & (Li et al., 2015b) & NWC & $108.6^{\circ} \mathrm{N}, 34.6^{\circ} \mathrm{E}$ & 4 & 12.9 & 527 & Clay & 10.57 & $\mathrm{U}$ & $\mathrm{W}, \mathrm{M}$ & $\mathrm{D}$ & $\begin{array}{l}\text { Wheat- } \\
\text { Maize }\end{array}$ & $\mathrm{Na}$ & 407 & $\mathrm{Na}$ \\
\hline
\end{tabular}




\begin{tabular}{|c|c|c|c|c|c|c|c|c|c|c|c|c|c|c|c|}
\hline $27-2$ & (Li et al., 2015b) & NWC & $108.6^{\circ} \mathrm{N}, 34.6^{\circ} \mathrm{E}$ & 4 & 12.9 & 527 & Clay & 10.57 & $\mathrm{U}$ & $\mathrm{W}, \mathrm{M}$ & $\mathrm{D}$ & Wheat & $\mathrm{Na}$ & 407 & $\mathrm{Na}$ \\
\hline $27-3$ & (Li et al., 2015b) & NWC & $108.6^{\circ} \mathrm{N}, 34.6^{\circ} \mathrm{E}$ & 4 & 12.5 & 527 & Clay & 10.57 & $\mathrm{U}$ & $\mathrm{W}, \mathrm{M}$ & $\mathrm{D}$ & Maize & $\mathrm{Na}$ & 407 & $\mathrm{Na}$ \\
\hline $27-4$ & (Li et al., 2015b) & NWC & $108.6^{\circ} \mathrm{N}, 34.6^{\circ} \mathrm{E}$ & 4 & 12.5 & 527 & Clay & 10.57 & $\mathrm{U}$ & $\mathrm{W}, \mathrm{M}$ & $\mathrm{D}$ & Maize & $\mathrm{Na}$ & 407 & $\mathrm{Na}$ \\
\hline 28 & (Li et al., 2015a) & $\mathrm{NC}$ & $113.67^{\circ} \mathrm{N}, 34.79^{\circ} \mathrm{E}$ & 24 & 14.4 & 640 & Sandy loam & 6.15 & $\mathrm{U}$ & $\mathrm{W}, \mathrm{M}$ & $\mathrm{D}$ & $\begin{array}{l}\text { Wheat- } \\
\text { Maize }\end{array}$ & $\mathrm{Na}$ & 142 & $\mathrm{Na}$ \\
\hline $29-1$ & (Liao et al., 1998) & $\mathrm{SC}$ & $120.22^{\circ} \mathrm{N}, 33.43^{\circ} \mathrm{E}$ & 5 & 15.7 & 1050 & Clay & 20.19 & $\mathrm{P}-\mathrm{U}$ & $\mathrm{W}, \mathrm{R}$ & $\mathrm{D}$ & Rice & 0.95 & 240 & $\mathrm{Y}$ \\
\hline $29-2$ & (Liao et al., 1998) & $\mathrm{SC}$ & $120.22^{\circ} \mathrm{N}, 33.43^{\circ} \mathrm{E}$ & 5 & 15.7 & 1050 & Clay & 20.01 & P-U & $\mathrm{W}, \mathrm{R}$ & $\mathrm{D}$ & Rice & 1.89 & 240 & $\mathrm{Y}$ \\
\hline $29-3$ & (Liao et al., 2009) & $\mathrm{SC}$ & $113.34^{\circ} \mathrm{N}, 28.62^{\circ} \mathrm{E}$ & 27 & 17 & 1385 & Silt Loam & 20.13 & $\mathrm{P}$ & $\mathrm{R}$ & $\mathrm{D}$ & Rice & 0.71 & 230 & $\mathrm{Y}$ \\
\hline $29-4$ & (Liao et al., 2009) & $\mathrm{SC}$ & $113.34^{\circ} \mathrm{N}, 28.62^{\circ} \mathrm{E}$ & 27 & 17 & 1385 & Silt Loam & 20.13 & $\mathrm{P}$ & $\mathrm{R}$ & $\mathrm{D}$ & Rice & 0.71 & 230 & $\mathrm{Y}$ \\
\hline $30-1$ & (Lin et al., 1997) & $\mathrm{SC}$ & $120.27^{\circ} \mathrm{N}, 30.27^{\circ} \mathrm{E}$ & 3 & 14.8 & 1270 & Clay & 25.34 & $\mathrm{P}-\mathrm{U}$ & $\mathrm{W}, \mathrm{R}$ & $\mathrm{T}$ & Rice & 2.68 & 415 & $\mathrm{Y}$ \\
\hline $30-2$ & (Lin et al., 1997) & $\mathrm{SC}$ & $120.27^{\circ} \mathrm{N}, 30.27^{\circ} \mathrm{E}$ & 3 & 14.8 & 1270 & Clay & 25.34 & $\mathrm{P}-\mathrm{U}$ & $\mathrm{W}, \mathrm{R}$ & $\mathrm{T}$ & $\begin{array}{l}\text { Rice- } \\
\text { Wheat }\end{array}$ & 2.76 & 415 & $\mathrm{Y}$ \\
\hline 31 & (Liu et al., 2013) & NWC & $107.5^{\circ} \mathrm{N}, 35.27^{\circ} \mathrm{E}$ & 30 & 9.8 & 540 & Loam & 6.2 & $\mathrm{U}$ & $\mathrm{W}, \mathrm{M}$ & $\mathrm{S}$ & $\begin{array}{l}\text { Wheat- } \\
\text { Maize }\end{array}$ & 2.45 & 90 & $\mathrm{Na}$ \\
\hline $32-1$ & (Liu et al., 2017) & $\mathrm{SC}$ & $120.2^{\circ} \mathrm{N}, 30.3^{\circ} \mathrm{E}$ & 4 & 16 & 1550 & Clay & 9.11 & $\mathrm{U}$ & $\mathrm{R}$ & $\mathrm{S}$ & Wheat & $\mathrm{Na}$ & 303 & $\mathrm{Y}$ \\
\hline $32-2$ & (Liu et al., 2017) & $\mathrm{SC}$ & $120.2^{\circ} \mathrm{N}, 30.3^{\circ} \mathrm{E}$ & 4 & 16 & 1550 & Clay & 11.37 & $\mathrm{U}$ & $\mathrm{R}$ & $\mathrm{S}$ & Wheat & $\mathrm{Na}$ & 303 & $\mathrm{Y}$ \\
\hline $33-1$ & (Liu et al., 2014) & $\mathrm{SC}$ & $111.5^{\circ} \mathrm{N}, 28.92^{\circ} \mathrm{E}$ & 21 & 17.2 & 1450 & Clay & 15 & $\mathrm{P}$ & $\mathrm{R}$ & $\mathrm{D}$ & Rice & 2.14 & 218 & $\mathrm{Y}$ \\
\hline $33-2$ & (Liu et al., 2014) & $\mathrm{SC}$ & $111.52^{\circ} \mathrm{N}, 29.24^{\circ} \mathrm{E}$ & 21 & 17.2 & 1450 & Clay & 8.16 & P-U & $\mathrm{R}, \mathrm{M}$ & $\mathrm{D}$ & Rice & 1.87 & 137 & $\mathrm{Y}$ \\
\hline $33-3$ & (Liu et al., 2014) & $\mathrm{SC}$ & $111.52^{\circ} \mathrm{N}, 29.24^{\circ} \mathrm{E}$ & 21 & 17.2 & 1450 & Clay & 6.09 & $\mathrm{U}$ & $\mathrm{R}$ & $\mathrm{D}$ & Rice & 1.87 & 310 & $\mathrm{Na}$ \\
\hline $34-1$ & (Lou et al., 2011) & NEC & $119.47^{\circ} \mathrm{N}, 41.67^{\circ} \mathrm{E}$ & 6 & 6.5 & 450 & Silt Loam & 9.8 & $\mathrm{U}$ & M & $\mathrm{S}$ & Maize & 0.31 & 0 & $\mathrm{Na}$ \\
\hline $34-2$ & (Lou et al., 2011) & NEC & $119.47^{\circ} \mathrm{N}, 41.67^{\circ} \mathrm{E}$ & 6 & 6.5 & 450 & Silt Loam & 9.8 & $\mathrm{U}$ & M & $\mathrm{S}$ & Maize & 0.47 & 0 & $\mathrm{Na}$ \\
\hline $34-3$ & (Lou et al., 2011) & NEC & $119.47^{\circ} \mathrm{N}, 41.67^{\circ} \mathrm{E}$ & 6 & 6.5 & 450 & Silt Loam & 9.8 & $\mathrm{U}$ & M & $\mathrm{S}$ & Maize & 0.85 & 0 & $\mathrm{Na}$ \\
\hline $34-4$ & (Lou et al., 2011) & NEC & $119.47^{\circ} \mathrm{N}, 41.67^{\circ} \mathrm{E}$ & 6 & 6.5 & 450 & Silt Loam & 9.8 & $\mathrm{U}$ & M & $\mathrm{S}$ & Maize & 1.07 & 248 & $\mathrm{Na}$ \\
\hline $34-5$ & (Lou et al., 2011) & NEC & $119.47^{\circ} \mathrm{N}, 41.67^{\circ} \mathrm{E}$ & 6 & 6.5 & 450 & Silt Loam & 9.8 & $\mathrm{U}$ & M & $\mathrm{S}$ & Maize & 1.79 & 248 & $\mathrm{Na}$ \\
\hline $34-6$ & (Lou et al., 2011) & NEC & $119.47^{\circ} \mathrm{N}, 41.67^{\circ} \mathrm{E}$ & 6 & 6.5 & 450 & Silt Loam & 9.8 & $\mathrm{U}$ & $\mathrm{M}$ & $\mathrm{S}$ & Maize & 3.07 & 248 & $\mathrm{Na}$ \\
\hline $34-7$ & (Lou et al., 2011) & $\mathrm{NEC}$ & $119.47^{\circ} \mathrm{N}, 41.67^{\circ} \mathrm{E}$ & 6 & 6.5 & 450 & Silt Loam & 9.8 & $\mathrm{U}$ & $\mathrm{M}$ & $\mathrm{S}$ & Maize & 2.05 & 248 & $\mathrm{Na}$ \\
\hline $34-8$ & (Lou et al., 2011) & NEC & $119.47^{\circ} \mathrm{N}, 41.67^{\circ} \mathrm{E}$ & 6 & 6.5 & 450 & Silt Loam & 9.8 & $\mathrm{U}$ & $\mathrm{M}$ & $\mathrm{S}$ & Maize & 3.31 & 248 & $\mathrm{Na}$ \\
\hline $34-9$ & (Lou et al., 2011) & $\mathrm{NEC}$ & $119.47^{\circ} \mathrm{N}, 41.67^{\circ} \mathrm{E}$ & 6 & 6.5 & 450 & Silt Loam & 9.8 & $\mathrm{U}$ & $\mathrm{M}$ & $\mathrm{S}$ & Maize & 4.66 & 248 & $\mathrm{Na}$ \\
\hline $34-10$ & (Lou et al., 2011) & NEC & $119.47^{\circ} \mathrm{N}, 41.67^{\circ} \mathrm{E}$ & 11 & 6.5 & 450 & Silt Loam & 9.8 & $\mathrm{U}$ & $\mathrm{M}$ & $\mathrm{S}$ & Maize & 0.31 & 0 & $\mathrm{Y}$ \\
\hline $34-11$ & (Lou et al., 2011) & NEC & $119.47^{\circ} \mathrm{N}, 41.67^{\circ} \mathrm{E}$ & 11 & 6.5 & 450 & Silt Loam & 9.8 & $\mathrm{U}$ & $\mathrm{M}$ & $\mathrm{S}$ & Maize & 0.47 & 0 & $\mathrm{Y}$ \\
\hline $34-12$ & (Lou et al., 2011) & NEC & $119.47^{\circ} \mathrm{N}, 41.67^{\circ} \mathrm{E}$ & 11 & 6.5 & 450 & Silt Loam & 9.8 & $\mathrm{U}$ & $\mathrm{M}$ & $\mathrm{S}$ & Maize & 0.85 & 0 & $\mathrm{Y}$ \\
\hline $34-13$ & (Lou et al., 2011) & NEC & $119.47^{\circ} \mathrm{N}, 41.67^{\circ} \mathrm{E}$ & 11 & 6.5 & 450 & Silt Loam & 9.8 & $\mathrm{U}$ & $\mathrm{M}$ & $\mathrm{S}$ & Maize & 1.07 & 248 & $\mathrm{Y}$ \\
\hline $34-14$ & 2 & NEC & $119.47^{\circ} \mathrm{N}, 41.67^{\circ} \mathrm{E}$ & 11 & 6.5 & 450 & Silt Loam & 9.8 & $\mathrm{U}$ & $\mathrm{M}$ & $\mathrm{S}$ & Maize & 1.79 & 248 & $\mathrm{Y}$ \\
\hline $34-15$ & (Lou et al., 2011) & NEC & $119.47^{\circ} \mathrm{N}, 41.67^{\circ} \mathrm{E}$ & 11 & 6.5 & 450 & Silt Loam & 9.8 & $\mathrm{U}$ & M & $\mathrm{S}$ & Maize & 3.07 & 248 & $\mathrm{Y}$ \\
\hline
\end{tabular}




\begin{tabular}{|c|c|c|c|c|c|c|c|c|c|c|c|c|c|c|c|}
\hline $34-16$ & (Lou et al., 2011) & $\mathrm{NEC}$ & $119.47^{\circ} \mathrm{N}, 41.67^{\circ} \mathrm{E}$ & 11 & 6.5 & 450 & Silt Loam & 9.8 & $\mathrm{U}$ & M & $\mathrm{S}$ & Maize & 2.05 & 248 & $\mathrm{Y}$ \\
\hline $34-17$ & (Lou et al., 2011) & NEC & $119.47^{\circ} \mathrm{N}, 41.67^{\circ} \mathrm{E}$ & 11 & 6.5 & 450 & Silt Loam & 9.8 & $\mathrm{U}$ & $\mathrm{M}$ & $\mathrm{S}$ & Maize & 3.31 & 248 & $\mathrm{Y}$ \\
\hline $34-18$ & (Lou et al., 2011) & NEC & $119.47^{\circ} \mathrm{N}, 41.67^{\circ} \mathrm{E}$ & 11 & 6.5 & 450 & Silt Loam & 9.8 & $\mathrm{U}$ & M & $\mathrm{S}$ & Maize & 4.66 & 248 & $\mathrm{Y}$ \\
\hline 35 & (Ma et al., 2008) & $\mathrm{SC}$ & $120.26^{\circ} \mathrm{N}, 31.28^{\circ} \mathrm{E}$ & 25 & 15.7 & 1094 & Loam & 14.04 & $\mathrm{P}-\mathrm{U}$ & $\mathrm{W}, \mathrm{R}$ & $\mathrm{D}$ & $\begin{array}{l}\text { Rice- } \\
\text { Wheat }\end{array}$ & 1.33 & 225 & $\mathrm{Na}$ \\
\hline 36 & (Ma et al., 1994) & $\mathrm{SC}$ & $117.48^{\circ} \mathrm{N}, 32.95^{\circ} \mathrm{E}$ & 4 & 15.5 & 1050 & Clay & 8.7 & $\mathrm{P}-\mathrm{U}$ & $\mathrm{W}, \mathrm{R}$ & $\mathrm{D}$ & Rice & 2.54 & 374 & $\mathrm{Na}$ \\
\hline 37 & $(\mathrm{Mu}, 2012)$ & NWC & $106.42^{\circ} \mathrm{N}, 35.4^{\circ} \mathrm{E}$ & 9 & 9.4 & 600 & Loam & 7.03 & $\mathrm{U}$ & $\mathrm{M}$ & $\mathrm{S}$ & Maize & 4.36 & 150 & $\mathrm{Y}$ \\
\hline 38 & (Pan et al., 2006) & $\mathrm{SC}$ & $120.79^{\circ} \mathrm{N}, 31.1^{\circ} \mathrm{E}$ & 17 & 16 & 1100 & Loam & 16.14 & $\mathrm{P}-\mathrm{U}$ & $\mathrm{R}$ & $\mathrm{D}$ & Rice & 0.76 & 428 & $\mathrm{Y}$ \\
\hline 39 & (Peng, 2006) & NEC & $126.48^{\circ} \mathrm{N}, 43.97^{\circ} \mathrm{E}$ & 15 & 5.6 & 562.8 & Clay & 13.24 & $\mathrm{U}$ & $\mathrm{R}, \mathrm{M}$ & $\mathrm{S}$ & Maize & 1.58 & 113 & $\mathrm{Y}$ \\
\hline $40-1$ & (Shi et al., 2017a) & $\mathrm{NC}$ & $113.1^{\circ} \mathrm{N}, 37.97^{\circ} \mathrm{E}$ & 24 & 7.3 & 454.7 & Loam & 14.21 & $\mathrm{U}$ & $\mathrm{M}$ & $\mathrm{S}$ & Maize & 1.26 & 150 & $\mathrm{Y}$ \\
\hline $40-2$ & (Shi et al., 2017a) & $\mathrm{NC}$ & $113.1^{\circ} \mathrm{N}, 37.97^{\circ} \mathrm{E}$ & 24 & 7.3 & 454.7 & Loam & 14.79 & $\mathrm{U}$ & $\mathrm{M}$ & $\mathrm{S}$ & Maize & 1.26 & 150 & $\mathrm{Y}$ \\
\hline 41 & (Shi et al., 2017b) & $\mathrm{NC}$ & $115.02^{\circ} \mathrm{N}, 36.87^{\circ} \mathrm{E}$ & 15 & 13.2 & 556 & Loam & 4.1 & $\mathrm{U}$ & $\mathrm{W}, \mathrm{M}$ & $\mathrm{D}$ & $\begin{array}{l}\text { Wheat- } \\
\text { Maize }\end{array}$ & $\mathrm{Na}$ & $\mathrm{Na}$ & $\mathrm{Na}$ \\
\hline 42 & (Song et al., 2007) & $\mathrm{NC}$ & $116.25^{\circ} \mathrm{N}, 40.21^{\circ} \mathrm{E}$ & 12 & 12 & 592 & Loam & 7.43 & $\mathrm{U}$ & $\mathrm{W}, \mathrm{M}$ & $\mathrm{D}$ & Maize & 1.34 & 300 & $\mathrm{Y}$ \\
\hline $43-1$ & (Su et al., 2012) & NWC & $100.35^{\circ} \mathrm{N}, 39.4^{\circ} \mathrm{E}$ & 4 & 7.6 & 116.8 & Silt Loam & 3.7 & $\mathrm{U}$ & $\mathrm{M}$ & $\mathrm{S}$ & Wheat & 1.4 & $\mathrm{Na}$ & $\mathrm{Na}$ \\
\hline $43-2$ & (Su et al., 2012) & NWC & $100.35^{\circ} \mathrm{N}, 39.4^{\circ} \mathrm{E}$ & 4 & 7.6 & 116.8 & Silt Loam & 3.7 & $\mathrm{U}$ & $\mathrm{M}$ & $\mathrm{S}$ & Wheat & 1.4 & $\mathrm{Na}$ & $\mathrm{Na}$ \\
\hline $44-1$ & (Liao et al., 1998) & NWC & $108.08^{\circ} \mathrm{N}, 34.3^{\circ} \mathrm{E}$ & 25 & 13 & 632 & Silt Loam & 8.1 & $\mathrm{U}$ & $\mathrm{W}, \mathrm{M}$ & $\mathrm{D}$ & Maize & 1.17 & 206 & $\mathrm{Na}$ \\
\hline $44-2$ & (Liao et al., 1998) & NWC & $108.08^{\circ} \mathrm{N}, 34.3^{\circ} \mathrm{E}$ & 25 & 13 & 632 & Silt Loam & 8.1 & $\mathrm{U}$ & $\mathrm{W}, \mathrm{M}$ & $\mathrm{D}$ & Maize & 2.33 & 206 & $\mathrm{Na}$ \\
\hline $44-3$ & (Liao et al., 1998) & NWC & $108.08^{\circ} \mathrm{N}, 34.3^{\circ} \mathrm{E}$ & 25 & 13 & 632 & Silt Loam & 8.1 & $\mathrm{U}$ & $\mathrm{W}, \mathrm{M}$ & $\mathrm{D}$ & Maize & 4.41 & 206 & $\mathrm{Na}$ \\
\hline 45 & (Sun et al., 2003) & $\mathrm{NC}$ & $116.25^{\circ} \mathrm{N}, 40.21^{\circ} \mathrm{E}$ & 12 & 12 & 592 & Loam & 7.14 & $\mathrm{U}$ & $\mathrm{W}, \mathrm{M}$ & $\mathrm{D}$ & Maize & 1.34 & 300 & $\mathrm{Na}$ \\
\hline $46-1$ & (Sun et al., 2007) & $\mathrm{SC}$ & $120.68^{\circ} \mathrm{N}, 31.46^{\circ} \mathrm{E}$ & 16 & 15.5 & 1038 & Clay & 19.78 & $\mathrm{P}-\mathrm{U}$ & $\mathrm{W}, \mathrm{R}$ & $\mathrm{D}$ & Rice & 0.95 & 180 & $\mathrm{Na}$ \\
\hline $46-2$ & (Sun et al., 2007) & $\mathrm{SC}$ & $120.68^{\circ} \mathrm{N}, 31.46^{\circ} \mathrm{E}$ & 16 & 15.5 & 1038 & Clay & 19.78 & $\mathrm{P}-\mathrm{U}$ & $\mathrm{W}, \mathrm{R}$ & $\mathrm{D}$ & Rice & 1.89 & 180 & $\mathrm{Na}$ \\
\hline $46-3$ & (Sun et al., 2007) & $\mathrm{SC}$ & $116.15^{\circ} \mathrm{N}, 28.32^{\circ} \mathrm{E}$ & 24 & 17.6 & 1795 & Clay & 16.19 & $\mathrm{P}-\mathrm{U}$ & $\mathrm{R}$ & $\mathrm{D}$ & Rice & 1.89 & 204 & $\mathrm{Na}$ \\
\hline $47-1$ & (Tan et al., 2007) & $\mathrm{NC}$ & $111.64^{\circ} \mathrm{N}, 36.15^{\circ} \mathrm{E}$ & 13 & 7.3 & 455 & Loam & 13 & $\mathrm{U}$ & $\mathrm{W}$ & $\mathrm{S}$ & $\mathrm{W}$ & $\mathrm{Na}$ & $\mathrm{Na}$ & $\mathrm{Y}$ \\
\hline $47-2$ & (Tan et al., 2007) & $\mathrm{NC}$ & $111.64^{\circ} \mathrm{N}, 36.15^{\circ} \mathrm{E}$ & 13 & 7.3 & 454.7 & Loam & 13 & $\mathrm{U}$ & $\mathrm{W}$ & $\mathrm{S}$ & $\mathrm{W}$ & $\mathrm{Na}$ & $\mathrm{Na}$ & $\mathrm{Y}$ \\
\hline $47-3$ & (Tan et al., 2007) & $\mathrm{NC}$ & $115.23^{\circ} \mathrm{N}, 38^{\circ} \mathrm{E}$ & 13 & 13 & 550 & Clay & 14 & $\mathrm{U}$ & $\mathrm{W}, \mathrm{M}$ & $\mathrm{D}$ & $\mathrm{W}$ & $\mathrm{Na}$ & $\mathrm{Na}$ & $\mathrm{Y}$ \\
\hline $47-4$ & (Tan et al., 2007) & $\mathrm{NC}$ & $115.23^{\circ} \mathrm{N}, 38^{\circ} \mathrm{E}$ & 13 & 13 & 550 & Clay & 14 & $\mathrm{U}$ & $\mathrm{W}, \mathrm{M}$ & $\mathrm{D}$ & $\mathrm{W}$ & $\mathrm{Na}$ & $\mathrm{Na}$ & $\mathrm{Y}$ \\
\hline $48-1$ & (Gao et al., 2006) & NWC & $108.08^{\circ} \mathrm{N}, 34.3^{\circ} \mathrm{E}$ & 25 & 12.9 & 632 & Silt Loam & 8.12 & $\mathrm{U}$ & $\mathrm{W}, \mathrm{M}$ & $\mathrm{D}$ & Maize & 1.17 & 207 & $\mathrm{Y}$ \\
\hline $48-2$ & (Gao et al., 2006) & NWC & $108.08^{\circ} \mathrm{N}, 34.3^{\circ} \mathrm{E}$ & 25 & 12.9 & 632 & Silt Loam & 8.12 & $\mathrm{U}$ & $\mathrm{W}, \mathrm{M}$ & $\mathrm{D}$ & Maize & 2.33 & 207 & $\mathrm{Y}$ \\
\hline $48-3$ & (Gao et al., 2006) & NWC & $108.08^{\circ} \mathrm{N}, 34.3^{\circ} \mathrm{E}$ & 25 & 12.9 & 632 & Silt Loam & 8.12 & $\mathrm{U}$ & $\mathrm{W}, \mathrm{M}$ & $\mathrm{D}$ & Maize & 4.65 & 207 & $\mathrm{Y}$ \\
\hline $49-1$ & (Wang et al., 1994) & $\mathrm{SC}$ & $121.4^{\circ} \mathrm{N}, 30.9^{\circ} \mathrm{E}$ & 15 & 17.6 & 1173.4 & Clay & 18.36 & $\mathrm{P}-\mathrm{U}$ & $\mathrm{W}, \mathrm{R}$ & $\mathrm{D}$ & $\begin{array}{l}\text { Rice- } \\
\text { Wheat }\end{array}$ & 1.24 & 0 & $\mathrm{Na}$ \\
\hline $49-2$ & (Wang et al., 1994) & $\mathrm{SC}$ & $121.4^{\circ} \mathrm{N}, 30.9^{\circ} \mathrm{E}$ & 15 & 17.6 & 1173.4 & Clay & 18.36 & $\mathrm{P}-\mathrm{U}$ & $\mathrm{W}, \mathrm{R}$ & $\mathrm{D}$ & $\begin{array}{l}\text { Rice- } \\
\text { Wheat }\end{array}$ & 1.24 & 360 & $\mathrm{Na}$ \\
\hline
\end{tabular}




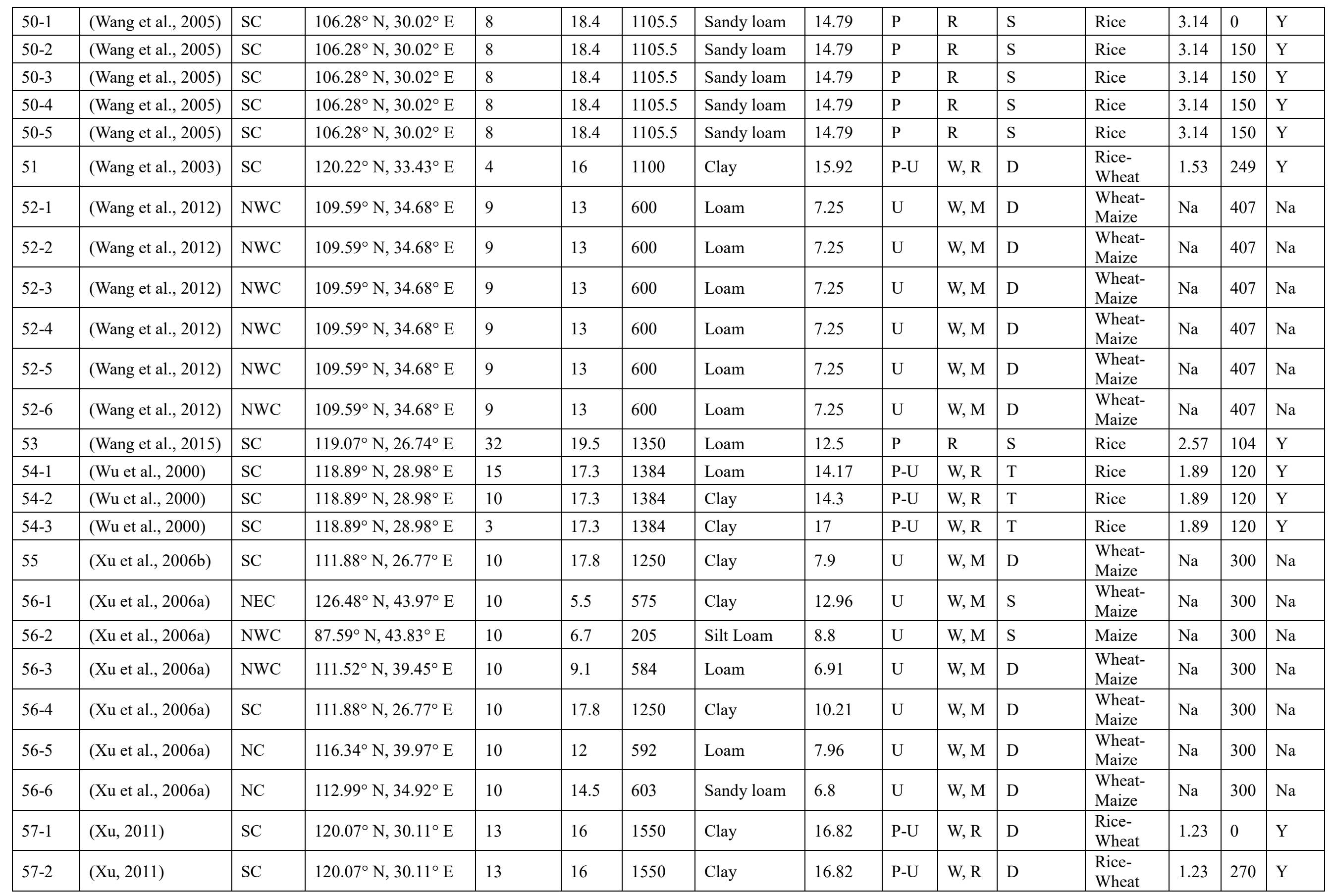




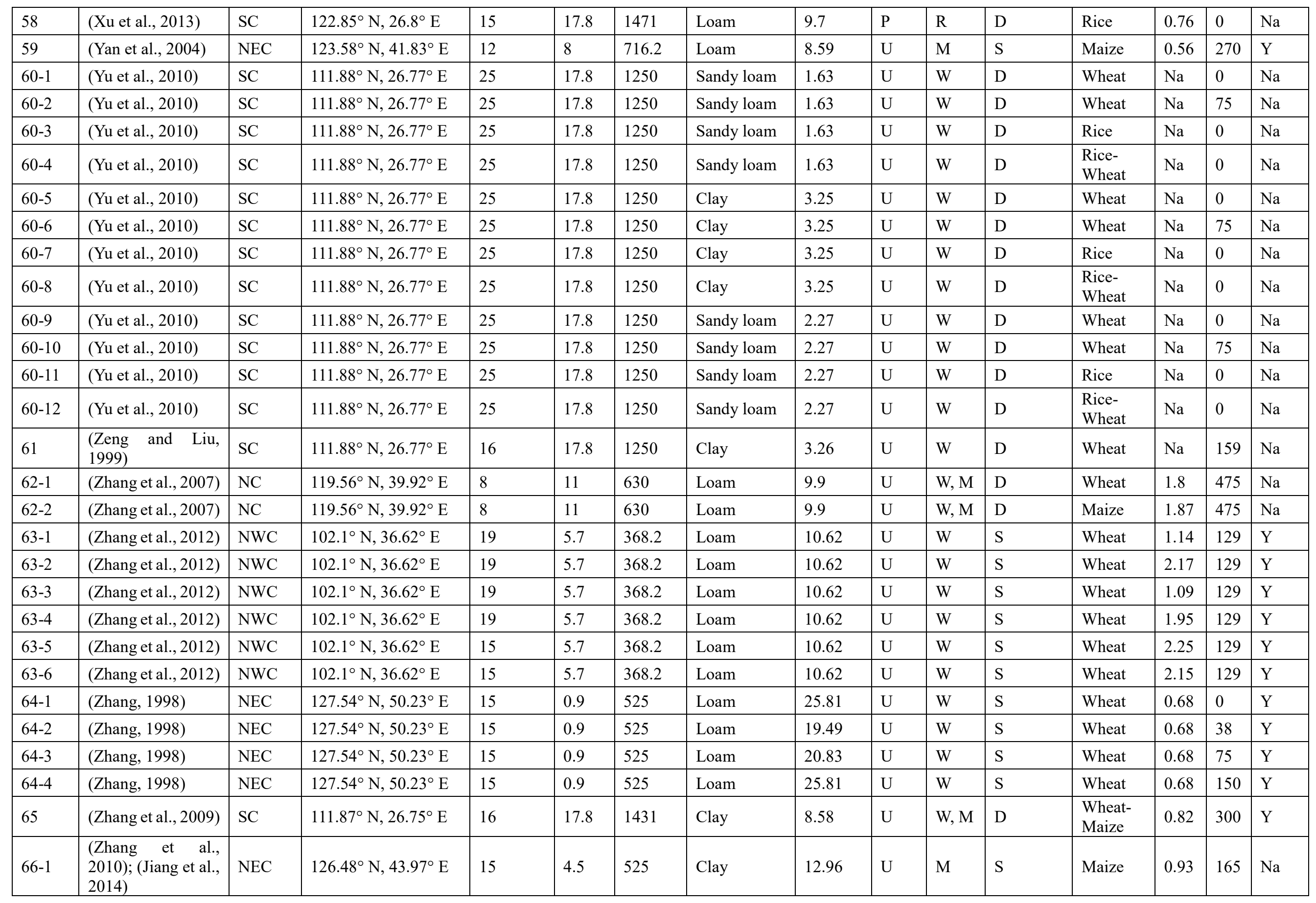




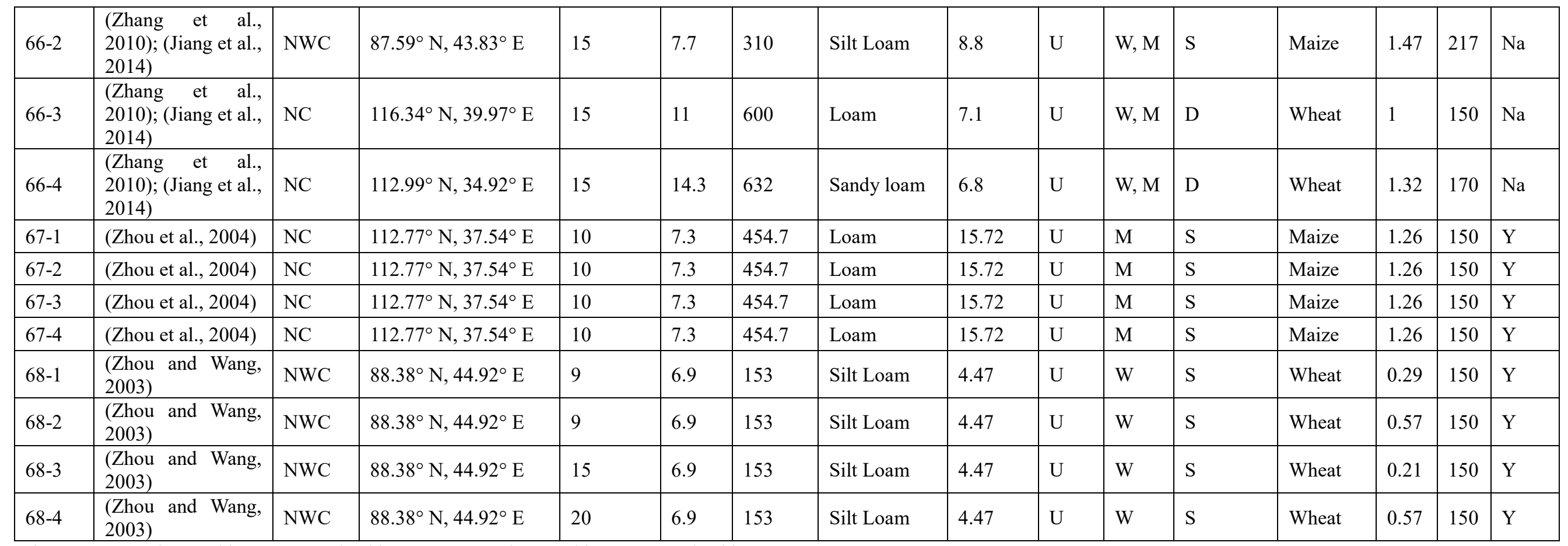

Region: NEC: Northeast China; NC: North China; NWC: Northwest China; SC: South China;

MAT: Mean annual temperature $\left({ }^{\circ} \mathrm{C}\right)$;

MAP: Mean annual precipitation ( $\mathrm{mm})$;

Land use: U: Upland soils; P: Paddy soils; P-U: Paddy-Upland soils;

5 Crop type: W: wheat; M: maize; R: rice;

Crop frequency (season $\mathrm{yr}^{-1}$ ): S: single cropping; D: double cropping; T: triple cropping;

STC: Straw-C input rate $\left(\mathrm{Mg} \mathrm{C} \mathrm{ha-1} \mathrm{yr}^{-1}\right)$;

$\mathrm{NF}$ : $\mathrm{N}$ fertilizer input rate $\left(\mathrm{kg} \mathrm{N} \mathrm{ha}^{-1} \mathrm{yr}^{-1}\right)$;

Y: yes; Na: no; 


\section{References}

Aiziguli-Mulati, Tong, Y. A., Yang, X. L., and Ma, H. Y.: Effect of different fertilization on soil organic carbon and its fraction in farmland, Chinese Journal of Soil Science, 43, 1461-1466, doi:10.19336/j.cnki.trtb.2012.06.032, 2012 (in Chinese).

5 Bi, L., Zhang, B., Liu, G., Li, Z., Liu, Y., Ye, C., Yu, X., Lai, T., Zhang, J., Yin, J., and Liang, Y.: Longterm effects of organic amendments on the rice yields for double rice cropping systems in subtropical China, Agr Ecosyst Environ, 129, 534-541, doi:10.1016/j.agee.2008.11.007, 2009.

Cai, T. Y., Huang, Y. W., Huang, H. J., Jia, Z. K., Li, L. K., Yang, B. P., and Han, S. M.: Soil labile organic carbon and carbon pool management index as affected by different years no-tilling with straw mulching, Chinese Journal of Ecology, 30, 1962-1968, doi:10.13292/j.1000-4890.2011.0328, 2011 (in Chinese).

Cao, Z. H., Zhu, Y. G., Liao, H. Q., and Wu, L. S.: Long-term expriment on soil nutrients balance and soil fertility improvement under the rice-wheat rotation in southern Jiangsu Provience, Soils, 2, 60 63+93, doi:10.13758/j.cnki.tr.1995.02.002, 1995 (in Chinese).

15 Chen, T., Du, L. J., Hao, X. H., Gao, J. Y., Luo, X. X., and Hu, R. G.: Effect of long-term fertilization on soil active organic carbon in paddy soils, Chinese Journal of Soil Science, 40, 809-814, 2009 (in Chinese).

Chen, W. R., Liu, Q., Yu, H. S., Wang, D. J., and Cao, Z. H.: Fractions and characteristics of dissolved organic matter in long-term fertilization paddy soils, Jouranl of Soil and Water Conservation, 24, 111-116, doi:10.13870/j.cnki.stbcxb.2010.06.026, 2010 (in Chinese).

Dong, B., Zhang, D. W., Guo, T. W., Zeng, J., and Bao, X. G.: Effect of long-term fertilization on soil organic carbon and microbial biomass carbon, Chinese Journal of Soil Science, 43, 1467-1472, doi:10.19336/j.cnki.trtb.2012.06.033, 2012 (in Chinese).

Dong, Z., Xie, H. T., Zhang, L. J., Bai, Z., He, H. B., Wang, G. M., and Zhang, X. D.: Effects of notillage practice with corn stalk mulching on soil properties in the Northeast of China, Journal of Maize Sciences, 21, 100-103+108, doi:10.13597/j.cnki.maize.science.2013.05.024, 2013 (in Chinese).

Fang, R. R., Tong, Y. A., Geng, Z. C., and Liang, D. L.: Effect of a long-term organic fertilization on wheat yield and soil fertility on loess plateau, Chinese journal of Eco-Agriculture, 11, 53-55, 2003 (in Chinese).

Gao, H.: Effects of straw returning methods on chernozem soil of soil respiration and carbon pool management index, Master, Northeast Agricultural University, Harbin, 48 pp., 2013 (in Chinese).

Gao, H. J., Peng, C., Zhang, X. Z., Li, Q., Zhu, P., and Fu, J. S.: Effect of long-term straw returning field on the carbon and nitrogen in black soil and maize yield, Journal of Maize Sciences, 19, 105-107, doi:10.13597/j.cnki.maize.science.2011.06.013, 2011 (in Chinese).

Gao, R., Lv, J. L., and Zhang, S., Xia.: Effect of long term fertilization on fertility of Lou soil and crop yield, Agriculture Research in the Arid Areas, 24, 64-67, doi:10.3321/j.issn:1000-7601.2006.05.015, 2006 (in Chinese).

Gao, X., Shen, A. L., Kou, C. L., Ma, Z. H., Wang, W. L., and Guo, Z. L.: Effects of straw application 
on soil organic carbon and active organic carbon in wheat-corn rotation system, Journal of Henan Agricultural Sciences, 41, 63-67, doi:10.15933/j.cnki.1004-3268.2012.09.008, 2012 (in Chinese).

Gao, Y. J., Zhu, P. L., Huang, D. M., Wang, Z. M., and Li, S. X.: Long-term impact of different soil management on organic matter and total nitrogen in rice-based cropping system, Soil and Environmental Sciences, 9, 27-30, doi:10.16258/j.cnki.1674-5906.2000.01.007, 2000 (in Chinese).

Gu, Q. Z., Yang, X. Y., Sun, B. H., Ma, L. J., Tong, Y. A., Zhao, B. Q., and Zhang, F. D.: Effects of longterm fertilization on soil nutrition and land productivity in topsoil of loess soil, Acta Agricultural Boreali-occident alis Sinica, 13, 121-125, doi:10.3969/j.issn.1004-1389.2004.03.032, 2004 (in Chinese).

Guan, Z. H., Li, Q. Y., Zhang, R. Z., Wang, L., and Zhang, J.: Effects of conservation tillage on readily oxidizable and total organic carbon in soil, Chinese Journal of Soil Science, 45, 420-426, 2014 (in Chinese).

Guo, J. H., Xing, Z., Li, C. J., and Han, B. W.: Study on the effects of fertilizer and straw return to soil on plowland Nutrients Variety, Journal of Hebei Agricultural Sciences, 7, 1-4, doi:10.3969/j.issn.1088-1631.2003.z1.001, 2003 (in Chinese).

Han, B., Kong, F. L., Zhang, H. L., and Chen, F.: Chinese Journal of Applied Ecology, Journal of Maize Sciences, 21, 91-98, doi:10.13287/j.1001-9332.2010.0065, 2010 (in Chinese).

Hao, X. X., Yang, C. B., Yuan, Y. R., Han, X. Z., Li, L. J., and Jiang, H.: Effects of continuous straw returning on organic carbon content in aggregates and fertility of black soil, Chinese Agricultual Science Bulletin, 29, 263-269, 2013 (in Chinese).

He, N. Z., and Ni, W. Z.: Fluctuation and balance of organic carbon in high-yielding paddy soil with wheat-rice-rice cropping system as affected by different fertilizer managment, Plant Nutrition and Fertilizer Science, 2, 315-321, 1996 (in Chinese).

Hou, Z. Y., Du, G. J., SUn, Z. X., Zheng, J. M., Yu, X. C., Hui, C. Z., Li, Z., and Wang, S. L.: Study on the effects of straw return to soil fertility, Rain Fed Crops, 24, 166-167, doi:10.3969/j.issn.20950896.2004.03.021, 2004 (in Chinese).

Huang, B. F.: Studies on effect of turnover of green-manure and wheat straw into soil on soil fertility, Acta Pedologica Sinica, 21, 113-122, 1984 (in Chinese).

Jiang, G., Xu, M., He, X., Zhang, W., Huang, S., Yang, X., Liu, H., Peng, C., Shirato, Y., and Iizumi, T.: Soil organic carbon sequestration in upland soils of northern China under variable fertilizer management and climate change scenarios, Global Biogeochem Cy, 28, 319-333, 2014.

Kong, H. M., and He, Y. Q.: Accumulation of organic matter and affecting factors in upland of red soil, Soils, 35, 401-407, doi:10.3321/j.issn:0253-9829.2003.05.008, 2003 (in Chinese).

Li, F., Xin, X. L., Zhang, C. Z., Ning, Q., Zhao, J. H., Wu, Q. C., Zhao, Z. H., Cai, T. Y., Nie, G. S., and

Zhang, J. B.: Soil Enzyme Levels in Fluvo-auic Soil with different long-term fertilization in North China Plain, Ecology and Environmental Sciences, 24, 984-991, doi:10.16258/j.cnki.16745906.2015.06.012, 2015a (in Chinese).

Li, K. J., Zhang, S., Fang., Song, P. Z., Liu, S., Qing., Huo, X. L., Wang, Y. D., and Jia, W. Z.: Effect of long-term fertilization on crop yield and soil fertility in semi-arid area Plant Nutrition and Fertilizer Science, 5, 21-25, doi:10.3321/j.issn:1008-505X.1999.01.004, 1999 (in Chinese). 
Li, K. J., Ma, J. Y., Cao, C. Y., Zheng, C. L., and SUn, W. F.: Effect of the long-term different organic fertilizer applications on crop yield and soil properties, Journal of Hebei Agricultural Sciences, 11, 60-63, doi:10.3969/j.issn.1088-1631.2007.01.021, 2007 (in Chinese).

Li, L., Hua., Z. H., Su, Y. R., Xiao, H. A., Huang, D. Y., and Wu, J. S.: Effects of rice straw incorporation in situ and ex situ on soil organic $\mathrm{C}$ and active organic $\mathrm{C}$ in agricultural soils in red soil hilly region, Scientia Agricultura Sinica, 42, 926-933, doi:10.3864/j.issn.0578-1752.2009.03.022, 2009 (in Chinese).

Li, S., Li, Y. B., Wang, S. J., Shi, J. L., and Tian, X. H.: Effects of different straw-returning regimes on soil organic carbon and carbon pool management index in Guanzhoong Plain, Northwest China, Chinese Journal of Applied Ecology, 26, 1215-1222, doi:10.13287/j.1001-9332.2015.0035, 2015b (in Chinese).

Li, W., Qiao, Y. Q., Chen, H., Du, S. Z., Zhao, Z., and Cao, C. F.: Carbon storages lime concretion black soils as affected by straw incorporation and fertilization, Ecology and Environmental Sciences, 23, 756-761, doi:10.3969/j.issn.1674-5906.2014.05.005, 2014 (in Chinese).

15 Li, X. A., Tong, C. L., Ping, J., Wu, J. S., and Wang, L. G.: Effects of long-term fertilization on soil organic matter and total nitrogen in paddy soil, Soils, 38, 298-303, doi:10.3321/j.issn:02539829.2006.03.011, 2006 (in Chinese).

Liao, H. Q., Shi, W. M., Yan, W. D., Wang, X. C., and Cao, Z. H.: The effect of combined application of organic and inorganic fertilizer on gleyic paddy soil fertility, Chinese Journal of Soil Science, 29, 9-10, 1998 (in Chinese).

Liao, Y. L., Zheng, S. X., Nie, J., Lu, Y. H., Xie, J., and Yang, Z. P.: Effects of Long-term application of fertilization and rice straw on soil fertility and sustainability of a reddish paddy soil productivity, Scientia Agricultura Sinica, 42, 3541-3550, doi:10.3864/j.issn.0578-1752.2009.10.020, 2009 (in Chinese).

Lin, X. Y., Zhang, Y. S., and He, N. Z.: Effects of straw application methods on crop yield and soil fertility in a triple cropping paddy soil, Journal of Zhejiang Agricultural University, 23, 47-50, 1997 (in Chinese).

Liu, E., Yan, C., Mei, X., Zhang, Y., and Fan, T.: Long-term effect of manure and fertilizer on soil organic carbon pools in dryland farming in northwest China, PLoS One, 8, e56536, doi:10.1371/journal.pone.0056536, 2013.

Liu, S., Huang, D., Chen, A., Wei, W., Brookes, P. C., Li, Y., and Wu, J.: Differential responses of crop yields and soil organic carbon stock to fertilization and rice straw incorporation in three cropping systems in the subtropics, Agr Ecosyst Environ, 184, 51-58, doi:10.1016/j.agee.2013.11.019, 2014.

Liu, X. X., Lu, R. H., Tao, Y. B., Wu, D. T., and Chen, Y. D.: Effects of straw application on improving soil fertility in different soil types, Journal of Zhejiang Agricultural Sciences, 58, 330-332+337, doi:10.1678/j.issn.0528-9017.20170243, 2017 (in Chinese).

Lou, Y., Xu, M., Wang, W., Sun, X., and Zhao, K.: Return rate of straw residue affects soil organic C sequestration by chemical fertilization, Soil Till Res, 113, 70-73, doi:http://dx.doi.org/10.1016/j.still.2011.01.007, 2011.

Ma, C. Z., Zhou, Q., and He, F.: Surplus-deficit distribution of organic carbon in soil under combined 
fertilization, Acta Pedologica Sinica, 31, 34-41, 1994 (in Chinese).

Ma, L., Yang, L. Z., Ci, E., Wang, Y., Yin, S. X., and Shen, M. X.: Humus composition and stable carbon isotope natural abundance in paddy soil under long-term fertilization, Chinese Journal of Applied Ecology, 19, 1951-1958, doi:10.13287/j.1001-9332.2008.0347, 2008 (in Chinese).

$\mathrm{Mu}, \mathrm{P} .:$ Response of integrated soil fertility and carbon sequestration to continuous entire maize stalk returning into field in loess plateau, PhD, Gansu Agricultural University, Gansu, 115 pp., 2012 (in Chinese).

Pan, G. X., Zhou, P., Zhang, X. H., Li, L. Q., Zheng, J. F., Qiu, D. S., and Chu, Q. H.: Effect of different fertilization practices on crop carbon assimilation and soil carbon sequestration: A case of a paddy under a long-term fertilization trial from the Tai Lake region, China, Acta Ecological Sinica, 26, 3704-3710, doi:10.3321/j.issn:1000-0933.2006.11.024, 2006 (in Chinese).

Peng, C.: Changes of organic carbon and nitrogen pool of black soil under long-term fertilization, Master, Chinese Academy of Agricultural Sciences, Beijing, 49 pp., 2006 (in Chinese).

Shi, K. J., Zhou, H. P., Xie, W. Y., Yang, Z. X., and Cheng, M.: Variation characteristics of readily oxidizable organic carbon and soil organic carbon pools under straw returning modes in cinnamon soil, Journal of Shanxi Agricultural Sciences, 45, 83-88, doi:10.3969/j.issn.1002-2481.2017.01.22, 2017a (in Chinese).

Shi, X. X., Zhao, Y., Zhang, L., Wu, W. L., and Meng, F. Q.: Effects of different agricultural practices on soil carbon pool in North China Plain, Environmental Science, 38, 301-308, doi:10.13227/j.hjkx.201605212, 2017b (in Chinese).

Song, Y. L., Tang, H. J., and Li, X. P.: The effects of long-term fertilization on crop yield and aquicinnamon soil organic matter, Acta Agricultural Boreali-Simica, 22, 100-105, 2007 (in Chinese).

Su, Y. Z., Yang, R., Yang, X., and Fan, G. P.: Effects of agricultural management practices on soil organic carbon and its fractions in newly cultivated sandy soil in Northwest China, Scientia Agricultura Sinica, 45, 2867-2876, doi:10.3864/j.issn.0578-1752.2012.14.009, 2012 (in Chinese).

Sun, R., Lian., Zhao, B. Q., Zhu, L. S., Xu, J., and Zhang, F. D.: Effects of long-term fertilization on soil enzyme activities and its role in adjusting-controlling soil fertility, Plant Nutrition and Fertilizer Science, 9, 406-410, doi:10.3321/j.issn:1008-505X.2003.04.005, 2003 (in Chinese).

Sun, X., Liu, Q., Wang, D. J., and Zhang, B.: Effect of long-term straw application on soil fertility, Soils, 39, 782-786, doi:10.13758/j.cnki.tr.2007.05.004, 2007 (in Chinese).

Tan, D. S., Jin, J. Y., Huang, S. W., Li, S. T., and He, P.: Effect of Long-term application of K fertilizaer and wheat straw to soil on crop yield and soil $\mathrm{K}$ under different planting systems, Scientia Agricultura Sinica, 40, 133-139, doi:10.3321/j.issn:0578-1752.2007.01.018, 2007 (in Chinese).

Wang, F., Li, Q. H., Lin, C., Zhong, S. J., He, C. M., and Li, Y. J.: Effect of different fertilization modes on topsoil organic carbon sequestration and productivity in yellow paddy field of southern China, Journal of Plant Nutrition and Fertilizer Science, 21, 1447-1454, doi:10.11674/zwyf.2015.0609, 2015 (in Chinese).

Wang, X. D., Shi, X. J., and Song, G. Y.: Effects of long-term rice straw returning on the fertility and productivity of purplish paddy soil, Plant Nutrition and Fertilizer Science, 11, 302-307, doi:10.3321/j.issn:1008-505X.2005.03.003, 2005 (in Chinese). 
Wang, Y., Ji, Q., Liu, S., Sun, H. Y., and Wang, X. D.: Effects of tillage practices on water-stable aggregation and aggregate-associated organic C in soils, Journal of Agro-Environment Science, 31, 1365-1373, 2012 (in Chinese).

Wang, Y. H., Ke, F. Y., Zhang, M. Z., Gu, Y. M., and Chen, C. H.: Research on the combined effect of long-term straw incorporation, Chinese Journal of Soil Science, 25, 53-56, doi:10.19336/j.cnki.trtb.1994.s1.016, 1994 (in Chinese).

Wang, Z. Z., Wu, J. M., Chen, L. G., and Zhu, P. P.: Effects of direct and whole straw manuring method on increasing yield of crop and fertility of soil in rice-wheat double cropping area of Taihu Lake district, Jiangsu Journal of Agricultural Science, 19, 151-156, doi:10.3969/j.issn.10004440.2003.03.005, 2003 (in Chinese).

Wu, H. H., Zhang, L. J., Zhang, H. Y., and Dong, Q. H.: Effects of different fertilization on crop yield and soil fertility in reddish paddy soil, Chinese Journal of Soil Science, 31, 125-126, doi:10.3321/j.issn:0564-3945.2000.03.010, 2000 (in Chinese).

Xu, L. L., Wang, Q. B., Zhang, X. Y., Sun, X. M., Dai, X. Q., Yang, F. T., Bu, J. F., and Wang, H. M.: Effects of applying different kind fertilizers on enzyme activities related to carbon, nitrogen, and phosphorus cycles in reddish paddy soil, Chinese Journal of Applied Ecology, 24, 909-914, doi:10.13287/j.1001-9332.2013.0244, 2013 (in Chinese).

Xu, M. G., Yu, R., Sun, X. F., Liu, H., Wang, B. R., and Li, J. M.: Effects of long-term fertilization on labile organic matter and carbon management index (CMI) of the typical soils of China, Plant Nutrition and Fertilizer Science, 12, 459-465, doi:10.3321/j.issn:1008-505X.2006.04.001, 2006a (in Chinese).

Xu, M. G., Yu, R., and Wang, B. R.: Labile organic matter and carbon management indes in red soil under long-term fertilization, Acta Pedologica Sinica, 43, 723-729, doi:10.3321/j.issn:05643929.2006.05.003, 2006b (in Chinese).

$\mathrm{Xu}, \mathrm{Z}$. X.: Influence of consecutive 13-year long-term fertilization on yields of rice and wheat and soil fertility in Xihu Plain, Plant Nutrition and Fertilizer Science, 17, 16-21, 2011 (in Chinese).

Yan, L., Song, Y., He, J., Chen, Y., Zhang, Y., Bao, Y. Y., and Guan, L. Z.: Effects of maize stems returning back to the field on the yield of plants and soil fertility, Chinese Journal of Soil Science, 35, 143 148, 2004 (in Chinese).

30 Yu, H. Q., Sun, N., Lv, J. L., Gao, J. S., Xu, M. G., and Wang, B. R.: Organic matter changes in three parent soils with different long-term fertilizations in red soil regions of southern China, Plant Nutrition and Fertilizer Science, 16, 92-98, 2010 (in Chinese).

Zeng, X. B., and Liu, G. L.: Effects of inorganic fertilizers and returning the crop straw into soil on adsorption-desorption of red soil, Soil and Environmental Sciences, 8, 45-49, doi:10.3969/j.issn.1674-5906.1999.01.013, 1999 (in Chinese).

Zhang, D. X., Han, Z. Q., Wang, Q. B., Chen, H. B., Chang, L. S., Yu, Y. Q., Liu, D. Q., and Wang, J. Y.: Dynamic change of soil organic matter quality as affected by different long-term fertilization treatments, Chinese Journal of Soil Science, 38, 251-255, doi:10.3321/j.issn:05643945.2007.02.010, 2007 (in Chinese).

Zhang, W., Xu, M., Wang, B., and Wang, X.: Soil organic carbon, total nitrogen and grain yields under 
long-term fertilizations in the upland red soil of southern China, Nutr Cycl Agroecosys, 84, 59-69, 2009.

Zhang, W. J., Wang, X. J., Xu, M. G., Huang, S. M., Liu, H., and Peng, C.: Soil organic carbon dynamics under long-term fertilizations in arable land of northern China, Biogeosciences Discussions, 6, 6539-6577, 2010 (in Chinese).

Zhang, Y. L., Lv, J. L., Jin, J. Y., Li, S. T., Chen, Z. Q., and Gao, X. S.: Effects of chemical fertilizer and straw return on soil fertility and spring wheat quality, Plant Nutrition and Fertilizer Science, 18, 307-314, 2012 (in Chinese).

Zhang, Z. J.: Effects of long-term wheat straw return on crop yield and soil fertility, Chinese Journal of Soil Science, 29, 11-12+14, doi:10.19336/j.cnki.trtb.1998.04.004, 1998 (in Chinese).

Zhou, B., and Wang, Z. Q.: Effects of long-term located fertilization on soil nutrient in the farmland ecosystems of grey desert soil oases, Agricultural Research in the Arid Areas, 21, 35-39, doi:10.3321/j.issn:1000-7601.2003.04.009, 2003 (in Chinese).

Zhou, H. P., Yang, Z. P., Li, H. M., and Guan, C. L.: Effect of straw return to field and fertilization in autumn on dryland corn growth and on water and fertilizer efficiency, Chinese Journal of Applied Ecology, 15, 1231-1235, doi:10.13287/j.1001-9332.2004.0261, 2004 (in Chinese). 